\title{
Erratum to: Diagnostic performance of tomosynthesis and breast ultrasonography in women with dense breasts: a prospective comparison study
}

\author{
Won Hwa Kim ${ }^{1,2} \cdot$ Jung Min Chang ${ }^{1}$ Joongyub Lee ${ }^{3}$ A Jung Chu ${ }^{4}$. \\ Mirinae Seo ${ }^{5}$ Hye Mi Gweon ${ }^{6}$ Hye Ryoung $\mathrm{Koo}^{7} \cdot \mathrm{Su}_{\text {Hyun Lee }}{ }^{1}$. \\ Nariya $\mathrm{Cho}^{1} \cdot$ Min Sun Bae ${ }^{1} \cdot$ Sung Ui Shin ${ }^{1} \cdot$ Sung Eun Song ${ }^{8}$. \\ Woo Kyung Moon ${ }^{1}$
}

Published online: 24 February 2017

(C) Springer Science+Business Media New York 2017

\section{Erratum to: Breast Cancer Res Treat (2017) 162:85-94 \\ DOI 10.1007/s10549-017-4105-z}

Unfortunately, in the original publication of the article, the study period was erroneously published as the study was performed between June 2012 and February 2014 (p. 86).
The correct study period is between June 2013 and June 2014.
The online version of the original article can be found under doi:10.1007/s10549-017-4105-z.

Jung Min Chang

imchangjm@gmail.com

1 Department of Radiology, Seoul National University Hospital, 101, Daehak-ro, Jongno-gu, Seoul 110-744, South Korea

2 Department of Radiology, Kyungpook National University Medical Center, Daegu, South Korea

3 Division of Clinical Epidemiology, Medical Research Collaborating Center, Biomedical Research Institution, Seoul National University Hospital, Seoul, South Korea

4 Department of Radiology, SMG-SNU Boramae Medical Center, Seoul National University College of Medicine, Seoul, South Korea

5 Department of Radiology, Kyung Hee University Hospital, Seoul, South Korea

6 Department of Radiology, Gangnam Severance Hospital, Yonsei University College of Medicine, Seoul, South Korea

7 Department of Radiology, Hanyang University College of Medicine, Seoul, South Korea

8 Department of Radiology, Korea University Anam Hospital, Korea University College of Medicine, Seoul, South Korea 